\title{
Controllable Preparation and Characterization of Silver Nanoparticles
}

\author{
Yi Zonghui ${ }^{1,2, *}$, Cao Jiangping ${ }^{1,2}$ \\ ${ }^{1}$ College of Chemistry and Chemical Engineering, Ningxia Normal University, Guyuan 756000, China; \\ ${ }^{2}$ Engineering and Technology Research Center of Liupanshan Resources, Ningxia Normal University, \\ Guyuan 756000, China.
}

Keywords: nano, silver, UV Vis absorption spectra

\begin{abstract}
Nano silver materials were prepared by using PVP as dispersant and hydrazine hydrate as reducing agent under the protection of inert gas. Nano silver materials with different particle sizes and morphologies were obtained by controlling the concentration of AgNO3. Different shapes and sizes of silver nanoparticles have different UV Vis absorption spectra. The method is simple in operation, short in reaction time, requiring less equipment and low preparation cost, and can be synthesized in one step under mild conditions.
\end{abstract}

\section{Preface}

Silver nanoparticles are widely used in electronics industry, chemical industry and medical science because of their excellent electrical conductivity, thermal conductivity, high oxidation resistance and bactericidal effect. The synthesis and assembly of silver nanoparticles or clusters is the basis for the development of nano silver materials.

However, the traditional method of preparation of silver nanoparticles, harsh reaction conditions, long reaction time, high equipment requirements, the preparation method is tedious, high preparation cost, but also frequently used toxic organic solvents, affect human health, causing environmental pollution, does not conform to the concept of green chemistry. Therefore, the development of simple, low-cost, batch preparation, rapid preparation of nano silver technology is particularly important.

\section{Part of the experiment}

\subsection{Chemical reagents}

The polyvinylpyrrolidone PVP used in this experiment was pure, silver nitrate, pure, hydrazine hydrate (85\%), pure solvent, deionized water, acetone (analytical pure).

\subsection{Preparation of materials}

Take 0.2 g PVP (K30, average molecular weight of 40000) dissolved in $200 \mathrm{~mL}$ deionized water, stirring, dissolved. The appropriate amount of silver nitrate is dissolved in the above $200 \mathrm{~mL}$ PVP water solution, stirred and dissolved, and the N2 in the 20 minutes is pre sent to drive away the air in the solution. The appropriate concentration is $85 \%$ hydrazine hydrate dissolved in $2 \mathrm{~mL}$ deionized water, and the solution of different concentration of reducing agent is obtained. Under the protection of $\mathrm{N} 2$ gas, the reduced reductant solution was rapidly added to silver nitrate solution, stirred for $10 \mathrm{~min}$, and then the nano silver material was obtained. The aqueous solution containing nano silver was centrifuged, washed with deionized water for 3 times, washed with acetone for 2 times, and then freeze-dried to obtain the purified silver nanoparticles. The reaction temperature was 25 , and the reaction time was $10 \mathrm{~min}$.

\subsection{Analysis and detection}

$\mathrm{X}$ ray powder diffraction (XRD) using Bruker D8 Advance $\mathrm{X}$ ray diffraction, Cu-K ray alpha 1, 1.54056 a wavelength, the scan rate of 3 degrees /min; using Merlin Compact field emission scanning electron microscopy ZEISS in Germany (SEM) to observe the surface morphology of 
materials; using UV-756 UV visible spectra of the factory in Shanghai branch spectrophotometer observation material characteristic absorption spectrum.

\section{Results and analysis}

The experimental conditions of several groups of experiments are shown in table 1.

Table 1. experimental conditions

\begin{tabular}{|c|c|c|c|c|}
\hline Experiment number & 1 & 2 & 3 & 4 \\
\hline AgNO3 concentration (mM) & 1.25 & 2.5 & 5.0 & 12.5 \\
\hline $\begin{array}{c}\text { Hydrazine hydrate } \\
\text { concentration (M) }\end{array}$ & 0.64 & 1.28 & 2.55 & 5.12 \\
\hline Ag+: hydrazine hydrate ratio & $1: 5$ & $1: 5$ & $1: 5$ & $1: 4$ \\
\hline
\end{tabular}

Fig. 1 is a XRD diffraction pattern of third sets of experimental products treated by freeze-drying. It can be seen from the figure 1 that all the diffraction peaks correspond to the characteristic diffraction peaks (JCPDS No. 04-0783) and Fm-3m (225) space groups of cubic silver. The diffraction peak of sharp, high strength, high crystallinity products. There is no impurity peak in the figure, indicating that the material is pure phase. 2 to 38.1degree angle of diffraction peak, 44.3 degrees, 64.4 degrees, 77.4 degrees respectively cubic silver [111], [200], [220] and [311] diffraction peaks. The diffraction peaks of [111], [200], [220] and [311] in Figure 1 are obviously broadened, indicating that the product has small particle size.

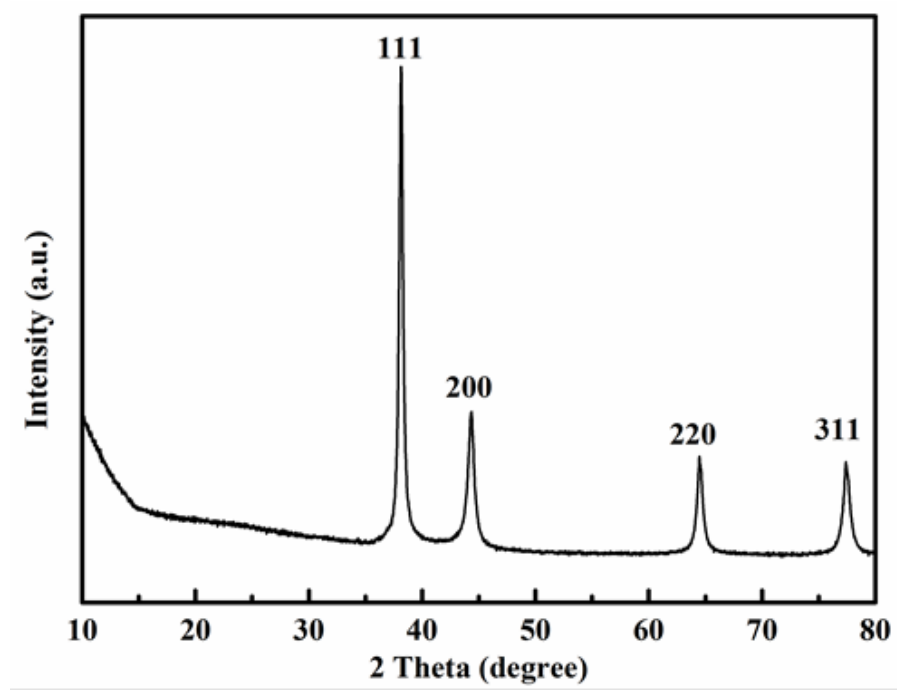

Figure 1. XRD diffraction pattern of the product

Figure 2 is the SEM diagram of the sample prepared by 4 sets of experiments. It can be seen from the figure 2A that when the concentration of silver nitrate is $1.25 \mathrm{mM}$, the product is fibrous, the average diameter of the nanofiber is about $60 \mathrm{~nm}$, and the length is about $2 \mathrm{um}$. Fig. $2 \mathrm{~b}$ is the product morphology when the concentration of silver nitrate is $2.5 \mathrm{mM}$. The silver in $2 \mathrm{~b}$ is nanoparticles, and a small amount of nanofibers are dispersed between the particles. 2C is a SEM with a concentration of $5 \mathrm{mM}$, and the product has good sphericity, narrow particle size distribution, and average particle size of about $80 \mathrm{~nm}$. Fig. 2D is SEM when the concentration of silver nitrate is $12.5 \mathrm{mM}$. The material synthesized in this condition is flaky nano silver, and the product has a serious aggregation phenomenon, probably because the concentration of PVP is low, and it can not sufficiently prevent the agglomeration between particles.

According to figure 2, when the concentration of silver nitrate is low, the synthesized product is nanofibers. With the increase of silver nitrate concentration, the nano silver gradually changes from fibrous to spherical, and when the concentration increases further, the sphere gradually changes into flake. 

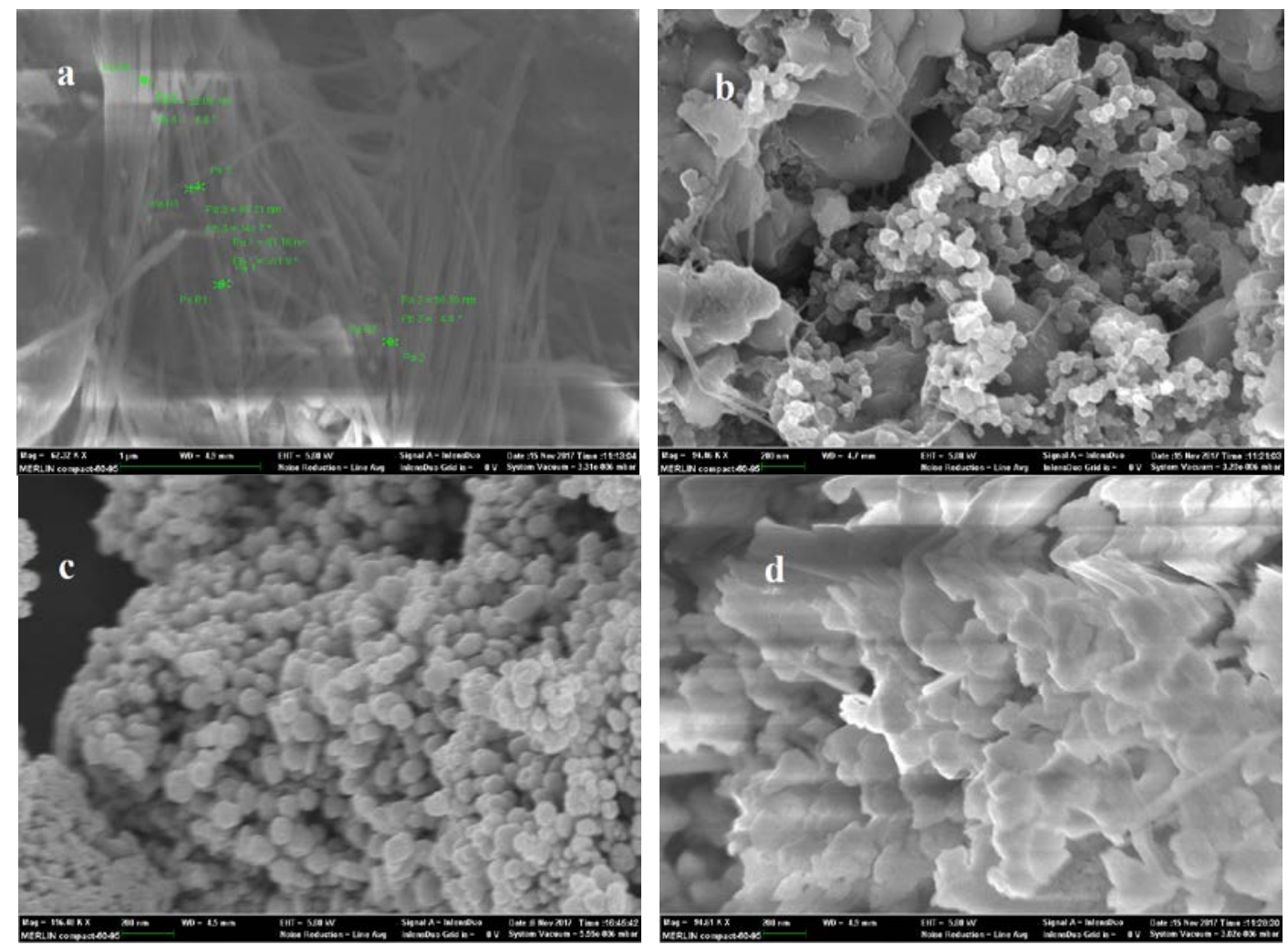

Figure 2 product SEM diagram: A, first groups of experiments SEM; B, second groups of experiments SEM; C, third groups of experiments SEM; D, fourth groups of experiments SEM.

Nanoparticles have many special properties, such as quantum size effect, surface effect, crystal field effect, so that the surface state of atoms, electrons and atoms, electrons in the internal behavior is different, resulting in nano particle has the same optical properties of new bulk materials are not available, mainly for broadband strong absorption, red shift and blue shift phenomenon.

Figure 3 is the UV Vis absorption spectra of the samples prepared by 4 sets of experiments. According to figure 3, when the concentration of silver nitrate is $1.25,2.5,5$ and $12.5 \mathrm{mM}$, the UV absorption peaks of products are 470, 400, 410 and $420 \mathrm{~nm}$, respectively. Therefore, UV silver nano fiber from the characteristic absorption peak is higher, and the ultraviolet absorption peaks of silver nanoparticles were compared with UV silver nano fiber with low absorption peaks, when the concentration of mM increased gradually from 2.5 to $12.5 \mathrm{mM}$, the $400 \mathrm{~nm}$ gradually shifted to high wave number move in the direction of ultraviolet absorption peak that, in the solution of silver nanoparticles size was gradually increased

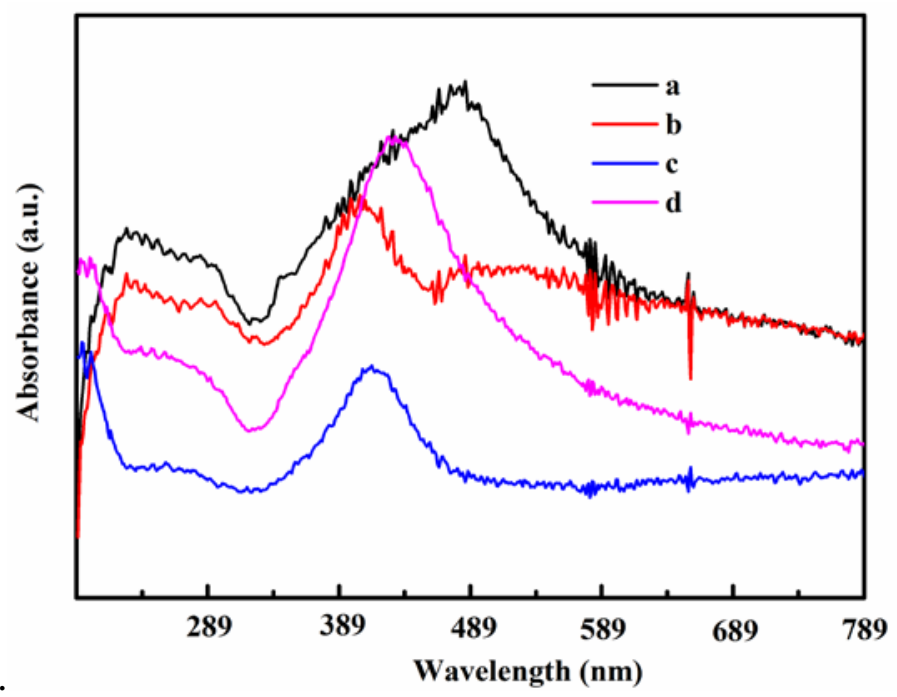

Fig. 3 UV Vis absorption spectra of products 


\section{Conclusion}

By controlling the concentration of silver nitrate, silver nanoparticles with different particle sizes and morphologies can be synthesized rapidly and conveniently. XRD test showed that the synthesized materials were single phase, cubic crystal system (Fm-3m space group), without impurities. By SEM, it was found that when the concentration of silver nitrate was low, the synthesized product was nanofibers. With the increase of silver nitrate concentration, the nano silver gradually changed from fiber to sphere, and then the flake silver nanoparticles were obtained when the concentration was increased further. When the concentration of silver nitrate was 1.25, 2.5, 5 and $12.5 \mathrm{mM}$, the UV absorption peaks were 470, 400, 410 and $420 \mathrm{~nm}$, respectively.

\section{Acknowledgment}

Project supported by the the Ningxia Hui Autonomous Region Education Department, item No. NGY2014112.

\section{References}

[1] Z. Zhang, B. Zhao, L. Hu, PVP Protective Mechanism of Ultrafine Silver Powder Synthesized by Chemical Reduction Processes, J. Solid State Chem. 121 (1996) 105-110.

[2] Y.H. Ding, P. Zhang, Z.L. Long, Y. Jiang, J.R. Yi, F. Xu, Y.D. Zuo, the elastic module of Ag nanowires prepared from electrochemical deposition, J. Alloys Compd. 474 (2009) 105-110.

[3] K. E. Korte, S. E. Skrabalak, Y. N. Xia, Rapid synthesis of silver nanowires through a CuCl- or $\mathrm{CuCl}_{2}$-mediated polyol process, J. Mater. Chem. 18 (2008) 437-441.

[4] J. Helmlinger, M. Heise, M. Heggen, M. Ruck, M. Epple, a rapid, high-yield and large-scale synthesis of uniform spherical silver nanoparticles by a microwave-assisted polyol process, Rsc Adv., 5 (2015) 92144-92150.

* communication contact; Yi Hong, female, lecturer, research direction: chemical materials 